\title{
The orbit evolution of 32 plutinos over 100 million year
}

\author{
X.-S. Wan $^{1}$ and T.-Y. Huang ${ }^{1,2}$ \\ 1 Astronomy Department, Nanjing University, Nanjing 210093, PR China \\ 2 National Astronomical Observatories, Beijing, PR China
}

Received 27 October 2000 / Accepted 4 January 2001

\begin{abstract}
The orbits of thirty two plutinos that are presently in the 3:2 mean motion resonance with Neptune have been integrated numerically and accurately to $10^{8}$ years into the future. Fourteen of them are found in unstable orbits after encountering Neptune or Pluto. Six of eighteen plutinos with stable orbits are in the Kozai resonance or around its separatrix zone. No node to node, perihelion to perihelion secular resonance or the so called 1:1 super resonance are found.
\end{abstract}

Key words. Kuiper belt, Oort cloud - minor planets, asteroids

\section{Introduction}

Edgeworth (1949) and Kuiper (1951) independently suggested that there would exist a large number of minor bodies in the solar system beyond the orbit of Neptune, which are the remains of planetesimals during the period of formation of the solar system. Interest in their conjecture has increased greatly since the first object was discovered in this region by Jewitt \& Luu (1993) in 1992. Astronomers believe that these bodies would give us a better understanding of the origin and evolution of the solar system. This region is now known as the Kuiper belt. Fernandez (1980), Duncan et al. (1988) numerically simulated the orbital motion of Kuiper belt objects (KBOs) and pointed out that they might be a source of the Juipter-family short period comets. Duncan et al. (1995) explored their orbital stability. They numerically integrated the orbits of thousands of KBOs up to the age of the solar system. They found that the orbits of KBOs with very small eccentricities, $e$, and with semi-major axes, $a$, larger than $36 \mathrm{AU}$ are mostly stable with the $40<a<42$ AU region being exceptional due to the presence of secular resonances. They pointed out that for KBOs with larger $e$ and $35<a<42$ AU the low order mean motion resonances with Neptune play a key role on their orbital stability (for an extensive review and comment, see also Malhotra et al. 2000). Up to now nearly 100 observed KBOs have been observed at multiple oppositions and are presumed to have orbits that are reasonably reliable. For $a<42 \mathrm{AU}$, they are trapped into mean motion resonances and have large eccentricities; for $a>42 \mathrm{AU}$, their positions are not particularly

Send offprint requests to: T.-Y. Huang, e-mail: tyhuang@nju.edu.cn related to mean motion resonances and usually have small eccentricities. Of reliably determined orbits, over $30 \%$ are trapped in the 3:2 mean motion resonance and they are the so called plutinos (Jewitt et al. 1996). The dynamic structure of the 3:2 resonance has been explored extensively (for example, Morbidelli 1997) since Cohen \& Hubbard (1965) found that Pluto is in the 3:2 mean motion resonance with Neptune. Malhotra (1993) suggested that Pluto originated from a nearly circular and less inclined orbit and the migration of the major planets in the early stage of the solar system excited its eccentricity and inclination and put it in the 3:2 resonance. Malhotra (1995) pointed out that this theory predicts a large population of plutinos and other resonant KBOs. Yu \& Tremaine (1999) adopted a model of the outer planets migration to make a theoretical analysis and a numerical simulation on the stability of plutinos. They found that the orbit of a plutino is stable when its eccentricity is either near that of Pluto $\left(\left|e-e_{\mathrm{P}}\right| / e_{\mathrm{P}} \lesssim 0.1\right.$, where $e$ and $e_{\mathrm{P}}$ are the eccentricities of the plutino and Pluto respectively) or very different from that of Pluto $\left(\left|e-e_{\mathrm{P}}\right| / e_{\mathrm{P}} \gtrsim 0.3\right)$.

For Pluto, there are two more resonances besides the 3:2 mean motion resonance. They are the Kozai resonance (Williams \& Benson 1971), which keeps its perihelion argument, $\omega$, librating around $90^{\circ}$, and the so called 1:1 super resonance (Williams \& Benson 1971; Milani et al. 1989; Wan et al. 2000), which keeps the libration period of $\omega$ close to the circulation period of the longitude difference between the ascending nodes of Pluto and Neptune, $\Omega-\Omega_{\mathrm{N}}$. Wan et al. (2000) gave the sizes of the stability zones of the three resonances in the space of orbital elements. Every resonance is a kind of protection mechanism to keep Pluto away from Neptune's pertubation. 
Table 1. Orbital elements of the five outer planets and the 32 plutinos at 2000.0 in the system of heliocentric invariant plane of 2000.0. An asterisk after a plutino's name marks its orbit based on only one opposition

\begin{tabular}{|c|c|c|c|c|c|c|c|}
\hline Object & $a(\mathrm{AU})$ & $e$ & $i^{\circ}$ & $\omega^{\circ}$ & $\Omega^{\circ}$ & $M^{\circ}$ & Source \\
\hline Jupiter & 5.20421 & 0.0488 & 0.3275 & 58.2257 & 317.3093 & 18.8574 & DE234 \\
\hline Saturn & 9.58195 & 0.0557 & 0.9296 & 325.7585 & 123.8796 & 320.3602 & DE234 \\
\hline Uranus & 19.22950 & 0.0444 & 1.0292 & 218.3694 & 312.2091 & 142.9217 & DE234 \\
\hline Neptune & 30.10557 & 0.0112 & 0.7244 & 202.1179 & 194.9391 & 268.1428 & DE234 \\
\hline Pluto & 39.26376 & 0.2447 & 15.5741 & 113.5018 & 110.5503 & 15.0162 & DE234 \\
\hline 1993RO & 39.87301 & 0.2100 & 3.2823 & 164.3786 & 195.4980 & 2.5474 & MPC 30688 \\
\hline 1993SB & 40.12059 & 0.3303 & 2.9354 & 107.1356 & 325.1308 & 328.4374 & MPC 36882 \\
\hline 1993SC & 40.12305 & 0.1915 & 5.9410 & 334.9581 & 340.5007 & 38.6813 & MPC 36882 \\
\hline 1994JR1 & 38.92671 & 0.1072 & 2.7134 & 82.7925 & 165.4456 & 4.6545 & MPC 34582 \\
\hline 1994TB & 40.24769 & 0.3287 & 13.5215 & 101.1591 & 314.1261 & 333.8047 & MPC 36884 \\
\hline 1995HM5 & 38.93880 & 0.2428 & 4.7966 & 42.8043 & 205.5407 & 327.3958 & MPC 32308 \\
\hline 1995QZ9 & 40.06416 & 0.1551 & 19.2446 & 141.9822 & 192.4231 & 31.9877 & MPC 30783 \\
\hline 1995YY3 & 39.97136 & 0.2319 & 1.1435 & 130.1496 & 290.9129 & 7.0493 & MPC 27920 \\
\hline 1996RR20 & 39.94925 & 0.1745 & 4.5878 & 35.5981 & 180.0305 & 114.3301 & MPC 30785 \\
\hline 1996ТР66 & 40.31454 & 0.3457 & 7.1108 & 81.5335 & 310.6128 & 359.4938 & MPC 36888 \\
\hline 1996TQ66 & 40.10226 & 0.1367 & 14.8730 & 26.4424 & 4.7160 & 0.9490 & MPC 36888 \\
\hline 1997QJ4 & 40.06144 & 0.2393 & 17.3723 & 84.7594 & 342.4586 & 316.8782 & MPC 36893 \\
\hline 1998НН151 & 38.44862 & 0.1546 & 8.8993 & 10.7611 & 204.9529 & 346.8681 & MPC 35440 \\
\hline 1998HK151 & 38.78986 & 0.2172 & 5.2893 & 193.3237 & 35.7187 & 3.0587 & MPC 34918 \\
\hline 1998HQ151 & 38.75965 & 0.2721 & 12.8919 & 340.4688 & 234.8507 & 10.4392 & MPC 34919 \\
\hline 1998UR43 & 40.14194 & 0.2278 & 7.8891 & 23.9622 & 44.7238 & 341.4225 & MPC 36820 \\
\hline 1998US43 & 40.04371 & 0.1517 & 11.3229 & 134.3818 & 230.9129 & 34.1429 & MPC 36379 \\
\hline 1998UU43 & 39.03955 & 0.2657 & 10.4695 & 257.3069 & 238.5366 & 293.6570 & MPC 36820 \\
\hline 1998VG44 & 39.84386 & 0.2584 & 1.6464 & 301.8364 & 147.0948 & 341.8865 & MPC 36821 \\
\hline 1998WV24 & 39.94157 & 0.1117 & 1.8881 & 240.9064 & 237.3031 & 299.2427 & MPC 36821 \\
\hline 1998WZ24* & 39.73140 & 0.1848 & 3.1866 & 8.6375 & 67.0976 & 1.9796 & MPC 35442 \\
\hline 1998WU31 & 40.12269 & 0.2088 & 7.6430 & 130.3469 & 246.3207 & 23.0274 & MPC 36821 \\
\hline 1998WV31 & 39.82698 & 0.2721 & 4.8126 & 291.5277 & 44.2743 & 40.0718 & MPC 36821 \\
\hline 1998WZ31* & 39.82047 & 0.1698 & 13.7894 & 11.5906 & 45.1206 & 1.8024 & MPC 33766 \\
\hline 1999JB132 & 39.25729 & 0.2744 & 13.5829 & 90.2935 & 218.2959 & 296.0702 & MPC 35470 \\
\hline 1999JC132* & 39.25828 & 0.2441 & 5.4625 & 297.7963 & 19.9211 & 284.1128 & MPC 35470 \\
\hline 1999JE132* & 39.18590 & 0.1944 & 25.5738 & 264.1790 & 216.3818 & 70.5880 & MPC 35470 \\
\hline 1999JK132* & 39.07611 & 0.1661 & 15.7368 & 87.4546 & 27.2109 & 80.3354 & MPC 35737 \\
\hline 1999KR16* & 39.08885 & 0.2274 & 28.6385 & 101.5305 & 208.4649 & 282.0770 & MPC 35737 \\
\hline $1999 \mathrm{KS} 16^{*}$ & 39.02739 & 0.2590 & 3.6820 & 320.7394 & 0.7318 & 273.9565 & MPC 35737 \\
\hline 1999TR11* & 40.03327 & 0.2520 & 15.9936 & 4.0646 & 50.3021 & 0.4365 & MPEC 1999-V05 \\
\hline 2000FE8* & 38.98400 & 0.0730 & 6.9935 & 193.1901 & 351.4466 & 0.7180 & MPEC 2000-G01 \\
\hline
\end{tabular}

Morbidelli et al. (1995) numerically followed the orbital evolution of five KBOs, including three plutinos, 1993RO, 1993SB and 1993SC. They found that the latter two are orbitally stable but the first one is in chaotic motion and will be expelled from the 3:2 resonance. Five years have passed since their computation. The orbits of these plutinos have been improved and more plutinos have been discovered. The significance of exploring the long term orbital evolution of plutinos is more than just the objects themselves; it also provides a good check on the theoretical dynamic structure of the 3:2 mean motion resonance. In this article we explore the present and future of plutinos by a long term numerical integration. Our numerical experiments will be described in Sect. 2 and our main results will be reported in Sect. 3 .

\section{Numerical experiments}

The model of solar system we adopt consists of the Sun and the five outer planets. The masses of the inner planets have been added to that of the Sun. The masses and the initial heliocentric coordinates and velocities of the 
outer planets in the mean equatorial system of 2000.0 are taken from DE234 (Standish 1993, private communication), with epoch June 28, 1969. The sources of the orbit elements of the 32 plutinos are listed in the last column of Table 1. The original elements are in the ecliptic coordinate system of 2000.0 and every plutino has its own epoch. Therefore we first transformed the coordinates and velocities of the outer planets from the mean equatorial system to the ecliptic system, then for each plutino we integrated the outer planets to its specified epoch. Finally, the five outer planets and the 32 plutinos were put into the same epoch 2000.0 and then we transformed the heliocentric ecliptic coordinate system to the heliocentric invariant plane coordinate system. The latter coordinate system consists of the invariant plane of the solar system in which the longitude zero is so chosen that the node of the ecliptic on the invariant plane have the same longitude in the two systems. The integrator for this procedure is a 12th order Cowell prediction-correction algorithm (PECE) with a step size of 10 days.

Table 1 lists the orbital elements of the five outer planets and the 32 plutinos at 2000.0 in the heliocentric invariant plane coordinate system of 2000.0, in which $a, e, i, \omega$, $\Omega$ and $M$ are the semi-major axis, eccentricity, inclination, perihelion argument, longitude of node and mean anomaly respectively. An asterisk marks orbits based on only one opposition.

We integrated the whole system for $10^{8}$ years starting from 2000.0. In order to ensure the accuracy of this long term integration we use a 12 th order symmetric method (Quinlan \& Tremaine 1990) as the integrator, which has similar advantages as symplectic integrators. The step size we adopt is 35 days and we save the data every 150000 steps for further data processing. A $10^{7}$ years run by the Cowell PECE integrator was taken as a comparison. This long term integration was run in double precision on a $440 \mathrm{MHz}$ SUN work station. The routine provided by Quinlan applies quadruple precision calculation at key operations in order to reduce the accumulation of roundoff errors.

\section{Main results}

We have found that the orbit evolution of the 32 plutinos can be classified into five categories: (a) The plutino is eventually expelled from the 3:2 resonance and falls inside the Hill radius of Neptune or Pluto. (b) The critical argument of the 3:2 mean motion resonance, $\sigma=3 \lambda-2 \lambda_{\mathrm{N}}-\varpi$, switches between libration and circulation, where $\lambda_{\mathrm{N}}$ and $\lambda$ are the mean longitudes of Neptune and the plutino respectively and $\varpi$ is the longitude of the perihelion of the plutino. (c) $\sigma$ is steadily in libration but no Kozai resonance is found. (d) $\sigma$ is steadily in libration and the perihelion argument, $\omega$, switches between libration and circulation. (e) Both $\sigma$ and $\omega$ are in libration.

Category (a) is the largest, which includes 14 plutinos, more than $40 \%$ of the total. The plutinos that would be expelled from the 3:2 resonance are listed in Table 2 .
Table 2. Plutinos in catagory (a), which encounter Neptune or Pluto

\begin{tabular}{lrr}
\hline & \multicolumn{2}{c}{ Time at encounter } \\
\cline { 2 - 3 } Plutino & with Neptune & with Pluto \\
\hline 1993SB & $9.5 \mathrm{Myr}$ & \\
1993SC & $4.87 \mathrm{Myr}$ & \\
1994JR1 & & $81.78 \mathrm{Myr}$ \\
1994TB & $30 \mathrm{Kyr}$ & \\
1995QZ9 & $26.76 \mathrm{Myr}$ & \\
1996RR20 & & $37.80 \mathrm{Myr}$ \\
1996TP66 & $70 \mathrm{Kyr}$ & \\
1997QJ4 & $29.72 \mathrm{Myr}$ & \\
1998HH151 & & $14 \mathrm{Kyr}$ \\
1998HK151 & & $3.15 \mathrm{Myr}$ \\
1998HQ151 & & $150 \mathrm{Kyr}$ \\
1998UU43 & $120 \mathrm{Kyr}$ & \\
1998WU31 & $18.85 \mathrm{Myr}$ & \\
2000FE8* & $30.12 \mathrm{Myr}$ & \\
\hline
\end{tabular}

In order to find the time of a sudden change of the semi-major axis of a plutino when it encounters Neptune or Pluto, we shortened the stepsize of our numerical integration. Table 2 lists the time of encounter with Neptune (Col. 2) or Pluto (Col. 3). To find the influence of Pluto's perturbation on the stability of the orbits of plutinos, we removed Pluto from our model and integrated the system again. We found that four plutinos, 1993SC, 1995QZ9, 1998WU31 and 2000FE8, now became stable and got well inside the $3: 2$ resonance, but five others, 1994JR1, 1996RR20, 1998HH151, 1998HK151 and 1998HQ151, would now encounter Neptune instead of Pluto and be expelled from the $3: 2$ resonance. This fact tells us that Pluto's perturbation has to be taken into account when computing orbits of plutinos. We would like to mention that most of former works (for example, Morbidelli et al. 1995; Duncan et al. 1995) did not include Pluto in their model.

Morbidelli et al. (1995) integrated the orbits of 1993RO, 1993SB and 1993SC for $500 \mathrm{Myr}$ and found that the orbits of the latter two are stable. The difference between their and our results in regard to these two bodies is mainly due to the improvement of the orbital elements in the mean time. The semi-major axis of 1993SB (Epoch 1994 Sep. 5.0) has been increased from 39.42 AU to 39.60 AU and that of 1993SC (Epoch 1995 Mar. 24.0) from 39.47 to 39.81 AU (see Table 1, Mobidelli et al. 1995). For using their model and initial condition and our program, we were able to find these two plutinos in regular orbits.

Category (b) consists of four members: 1996TQ66, 1998UR43, 1998US43 and 1999TR11. Their critical arguments in the 3:2 mean motion resonance switch between libration and circulation. Figure 1 shows the time variation of the semi-major axis $a$ and the critical argument $\sigma$ of 1998UR43. We can see that $a$ is well kept between 39 and $40 \mathrm{AU}$ and the maximum of $\sigma$ is greater than $300^{\circ}$, a signal of its proximity to chaos. These four plutinos should 

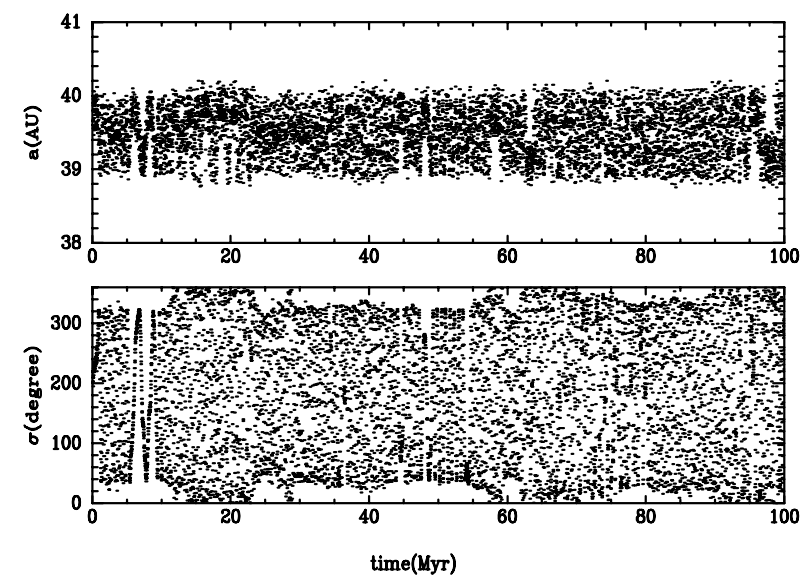

Fig. 1. Time variation of the semi-major axis $a$ and the critical argument $\sigma$ of 1998UR43

be regarded as being in the chaotic separatrix zone of the 3:2 resonance.

1993RO, 1995HM5, 1995YY3, 1998WV24, 1998WZ24, 1998WZ31, 1999JK132 and 1999KS16 fall into category (c). They remain in the 3:2 resonance during the whole of our integration and show no sign of the Kozai resonance. Special attention should be paid to 1993RO. Morbidelli et al. (1995) has shown that it is in strong chaos but Duncan et al. (1995) showed that it is stable for more than 1 Gyr with a set of improved orbit elements. In our integration 1993RO is also very stable and its $\sigma$ librates between $100^{\circ}$ and $260^{\circ}$. Its semi-major axis adopted by Morbidelli et al. (1995) is 39.696 AU at 1994 Sep. 5.0 and ours is $39.507 \mathrm{AU}$ at the same epoch. We did a $10^{8}$ years integration of $1993 \mathrm{RO}$ with their model and initial elements but with our symmetric integrator. We did not find any chaotic behavior either but its $\sigma$ librated between $70^{\circ}$ and $300^{\circ}$. We suspect that the difference was probably caused by their adopted integrator being less accurate. The maximum $300^{\circ}$ could be a signal that it is near the separatrix and an accurate integration would be necessary to trace its true evolution.

Malhotra (1996, Fig. 6) pointed out that there would exist two kinds of stable librators in the 3:2 Neptune resonance, aphelion librators and perihelion librators, according to it being near its aphelion or perihelion when closest to Neptune. All the plutinos in our categories (c), (d) and (e) are found to be aphelion librators. This is because their eccentricities are not large enough (see Table 1) and a large eccentricity would cause instability when more major planets besides Neptune are taken into consideration. We suspect that there are no realistic plutinos of the perihelion librator type.

Category (d) has only 2 members: 1998WV31 and 1999JC132. Their perihelion arguments, $\omega$, switch between libration and circulation but their critical arguments $\sigma$ are in libration. Consequently, they are always in the 3:2 mean motion resonance but come in and out of the separatrix region of the Kozai resonance. Figure 2a shows the $\omega$ of 1999JC132, which alternately librating around $90^{\circ}$ and $270^{\circ}$. Figure $2 \mathrm{~b}$ displays the orbit of $1998 \mathrm{WV} 31$ in the $(e, \omega)$ plane. It clearly shows that there exist two stable libration points at $\omega=90^{\circ}, 270^{\circ}$ and $e \sim 0.25$, and two unstable libration points at $\omega=0^{\circ}, 180^{\circ}$. $1998 \mathrm{WV} 31$ is obviously situated in a chaotic separatrix zone of the Kozai resonance. Since Fig. $2 b$ is a projection of a six dimensional orbit on a plane, one should expect a certain amount of dispersion of the points, but our above description is probably correct by the large area of the dispersion.

Catagory (e) contains objects inside the Kozai resonance. They are 4, namely, 1999JB132, 1999KR16, 1998WV24 and 1999JE132. The first two have their $\omega$ librating around $90^{\circ}$ and the last two around $270^{\circ}$. Compared to catagory (c), these four have smaller oscillation amplitude in semi-major axis, and larger oscillation amplitudes in eccentricity and inclination. Large oscillations in $e$ and $i$ excited by the Kozai resonance have been predicted by many authors, for example, Kozai (1962) and Thomas \& Morbidelli (1996). A larger $e$ would cause instability but a larger $i$ accompanied with the Kozai resonance could increase stability. In the case of Pluto and plutinos, it is evident that the Kozai resonance increases their orbit stability and plays the role of protection mechanism by reducing the perturbation of Neptune. Another dynamical property (Thomas \& Morbidelli 1996) that there should be a strong correlation between $e$ and $i$ for plutinos in the Kozai resonance can be clearly seen in Fig. 2c.

Pluto is in a third resonance, the so called 1:1 super resonance, as mentioned in the introduction section. We have not found any plutino in this resonance and believe that this protection mechanism is not important for orbital stability. Furthermore, we carefully checked all the possible secular resonances, perihelion to perihelion, node to node, but none of them were found.

In order to find the stable resonance zone in the $(a, e)$ plane and to compare our numerical result with the theoretical work of Morbidelli (1997, Fig. 8), we plot all the orbits of plutinos in our categories (b)-(e) in Fig. 3a, which outlines the 3:2 mean motion resonance zone. One can see that our distribution is smaller than Morbidelli's theoretical result from an averaged potential and in the limiting case of $i=0$, but is quite consistent with Malhotra's numerical result from a planar restricted 3-body model (Malhotra et al. 2000, Fig. 3). Figure 3b shows the plot for categories (d) and (e) only. One can see that the stable Kozai resonance zone is inside the 3:2 resonance and occupies a small region around $a=39.5 \mathrm{AU}$ and $e=0.25$. We did not find any example of Kozai resonance shown in the lower part (small eccentricity) of Fig. 8 of Morbidelli (1997).

\section{Discussion}

It is hard to believe that so many plutinos (14 of 32, nearly $44 \%$ ) are in unstable orbits (category (a)) after a long term evolution of the solar system. If this is true, the present KBOs do provide a rich source of short period comets. We suspect that the story will change when their orbits are 


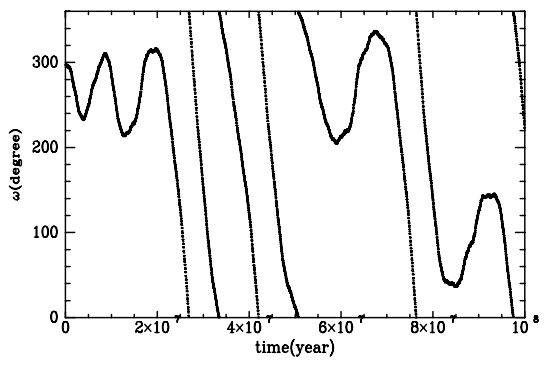

(a)

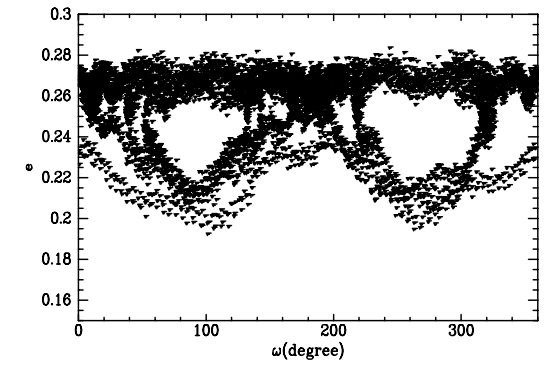

(b)

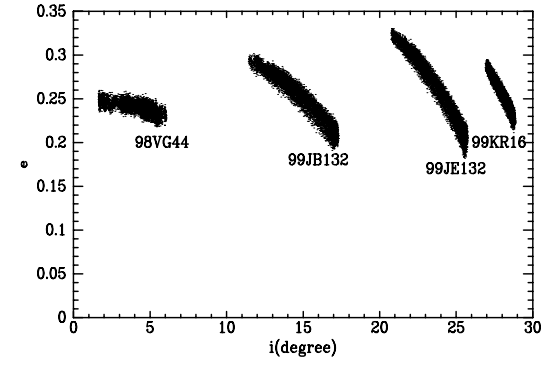

(c)

Fig. 2. a) Time variations of the perihelion argument $\omega$ of 1999JC132. b) Projection of the orbit of 1998 WV 31 on the $(e, \omega)$ plane. c) Correlation between $e$ and $i$ of the plutinos that are kept in the Kozai resonance

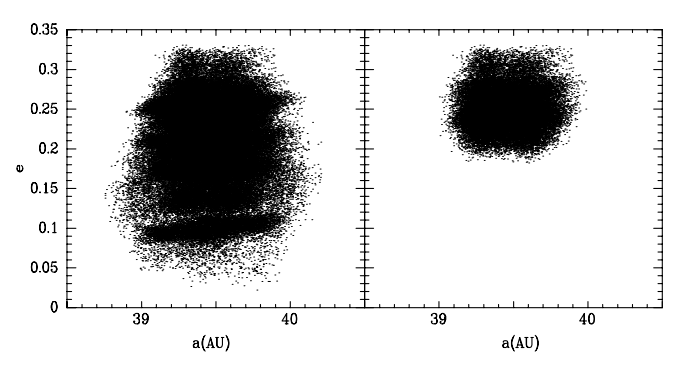

(a)

Fig. 3. a) Stable zone of the 3:2 mean motion resonance with Neptune from the orbit evolution of plutinos in catagories b) -e). b) Stable zone of the Kozai resonance from the orbit evolution of plutinos in catagories $\mathbf{d}$ ) and $\mathbf{e}$ )

more precisely determined, although among the 14 members in category (a) only 2000FE8's orbit was determined from a single opposition. The orbits of 1993SB and 1993SC have been determined from seven oppositions but they are unstable from our computation.

Pluto's gravitation plays an important role on plutinos' orbital stability. In the numerical experiments of many previous authors, Pluto's perturbation was not included. Our computation shows that Pluto's perturbation would cause instability, as in the case of 1993SC, 1995QZ9, 1998WU31 and 2000FE8. Moreover, no 1:1 mean motion resonance with Pluto has been found for the 32 plutinos we studied.

Of the 18 stable plutinos (categories (b)-(e)) 6 (in catagories (d) and (e)) are in the Kozai resonance or around its boundary. This is a quite large ratio. Further research on the Kozai resonance inside a mean motion resonance would be necessary. We were told that the behavior of the Kozai resonance depends on whether or not it is inside a mean motion resonance (Morbidelli 2000, private communication). A theoretical explanation of the behavior of 1998WV31 (Fig. 2b) should be worked out. The Kozai resonance zone of the six plutinos is confined to the upper part of the theoretical location worked out by Morbidelli (1997, Fig. 8). We do not understand why the Kozai resonance in nature happens only in a limited

region, much smaller than that predicted by Morbidelli's theory.

Pluto has three resonances to protect it from Neptune's perturbation. Up to now we have not found any plutino in the 1:1 super resonance. Why, then, is Pluto so peculiar? We now know that Pluto evolved into the 3:2 mean motion resonance by planets migration (Malhotra 1993). Malhotra (1995) also showed that Pluto and plutinos would be captured into the Kozai resonance with a certain probability due to planets migration. But Pluto's orbit is peculiar for the existence of the so called 1:1 super resonance and we know that the resonance zone of this super resonance is rather small (Wan et al. 2000). How did Pluto evolve into the 1:1 super resonance and why is it so different from the plutinos? This question should be addressed even though both the Kozai resonance and the 1:1 super resonance are not essential for Pluto's orbital stability (see Malhotra \& Williams 1997).

Two important issues regarding our numerical results on the orbital stability of known plutinos must be recognized and emphasized. First, the orbits of these objects are not very well determined considering that their orbital periods are in excess of 250 years but the observational arcs are only 2-7 years long. Secondly, the phase space in the vicinity of the $3: 2$ Neptune resonance is known to be very small and to contain a complex mix of stable and unstable orbits. In other words, orbital stability in this region is a very sensitive function of initial conditions. Consequently, the error bars on the orbital elements of the observed plutinos may well span a range of stable and unstable orbits. Thus, it is not clear that the present 32 plutino orbits are a fair and representative sample of the real population. The presently estimated KBO orbits (even the multi-opposition ones) are still not "reliable", and a need exists for an evaluation of the relationship between the sample of known orbits and the underlying real population.

Acknowledgements. We are grateful to Renu Maholtra for her helpful comments and suggestions. The last paragraph of the discussion section comes from her comments. We thank Tao Kiang for his help on English writing of this paper. This work is supported by the National Science Foundation of China 
under the project number 19633010 and the Minor Planets Foundation of the Purple Mountain Observatory.

\section{References}

Cohen, C. J., \& Hubbard, E. C. 1965, AJ, 70, 10

Edgeworth, K. E. 1949, MNRAS, 109, 600

Duncan, M., Quinn, T., \& Tremaine, S. 1988, AJ, 94, 1330

Duncan, M. J., Levison, H. F., \& Budd, S. M. 1995, AJ, 110, 3073

Fernandez, J. A. 1980, MNRAS, 192, 481

Jewitt, D., \& Luu, J. 1993, Nature, 362, 730

Jewitt, D., Luu, J., \& Chen, J. 1996, AJ, 112, 1225

Kozai, Y. 1962, AJ, 67, 591

Kuiper, G. 1951, On the origin of the solar system, in Astrophysics: A Topical Symposium, ed. J. A. Hynek (NY: McGraw Hill), 357
Malhotra, R. 1993, Nature, 365, 819

Malhotra, R. 1995, AJ, 110, 420

Malhotra, R. 1996, AJ, 111, 504

Malhotra, R., \& Williams, J. 1997, in Pluto and Charon, ed. D. J. Tholen, \& S. A. Stern (University of Arizona Press), 127.

Malhotra, R., Duncan, M., \& Levison, H. 2000, in Protostars and Planets IV, preprint

Milani, A., Nobili, A., \& Carpino, M. 1989, A\&A, 210, 313

Morbidelli, A., Thomas, F., \& Moons, M. 1995, Icarus, 118, 322

Morbidelli, A. 1997, Icarus, 127, 1

Quinlan, G. D., \& Tremaine, S. 1990, AJ, 100, 1694

Thomas, F., \& Morbidelli, A. 1996, CeMDA, 64, 209

Wan, X.-S., Huang, T.-Y., \& Innanen, K. A. 2001, AJ, in press Williams, J., \& Benson, G. S. 1971, AJ, 76, 167

Yu, Q., \& Tremaine, S. 1999, AJ, 118, 1873 